\title{
CORRECTION
}

Shunhu Zhang - Miao Guan • Guijing Wu - Siwei Gao •

Xiaodong Chen

\section{Correction to: An ellipsoidal yield criterion for porous metals with accurate descriptions of theoretical strength and Poisson's ratio}

Published online: 2 November 2017

(C) Springer-Verlag GmbH Austria 2017

\section{Correction to: Acta Mech \\ DOI 10.1007/s00707-017-1921-5}

In the original article, the authors' names are published incorrectly.

The correct authors' names are given below:

Shunhu Zhang, Miao Guan, Guijing Wu, Siwei Gao, Xiaodong Chen

The online version of the original article can be found under doi: 10.1007/s00707-017-1921-5.

S. Zhang $(\varangle) \cdot$ M. Guan $\cdot$ G. Wu $\cdot$ S. Gao

Shagang School of Iron and Steel, Soochow University, Suzhou 215021, China

E-mail: shzhang@suda.edu.cn 